\title{
Le Pérou: de l'État catholique à l'État laïque ou
} pluriconfessionnel?

L'institution du «Patronat» au Pérou

Marco Antonio Huaco Palomino

\section{(2) OpenEdition}

\section{Journals}

Édition électronique

URL : http://journals.openedition.org/assr/21252

DOI : $10.4000 /$ assr. 21252

ISSN : $1777-5825$

Éditeur

Éditions de l'EHESS

Édition imprimée

Date de publication : 1 juin 2009

Pagination : 99-118

ISBN : 978-2-7132-2216-0

ISSN : 0335-5985

\section{Référence électronique}

Marco Antonio Huaco Palomino, « Le Pérou: de l'État catholique à l'État laïque ou

pluriconfessionnel? », Archives de sciences sociales des religions [En ligne], 146 | avril-juin 2009, mis en ligne le 01 juillet 2012, consulté le 20 avril 2019. URL : http://journals.openedition.org/assr/21252 ; DOI : 10.4000/assr.21252 


\section{Marco Antonio Huaco Palomino}

\section{Le Pérou : de l'État catholique à l'État laïque ou pluriconfessionnel?}

\section{L'institution du " Patronat " au Pérou}

Au Pérou, l'histoire des relations entre l'Église et l'État ${ }^{1}$ a été principalement celle des relations avec l'Église catholique qui ont été régies successivement par le Patronat royal (1508-1821), le Patronat républicain de facto (1821-1875), le Patronat républicain de jure (1875-1979) et actuellement par le Concordat (1979 à nos jours).

Le Patronat est une institution juridique qui date de l'époque coloniale et qui lie l'État à l'Église par un pacte politique distribuant le pouvoir et les compétences séculaires et religieuses concernant le culte, la propagation de la foi, le contrôle social et la légitimation du pouvoir. Ce pacte pouvait prendre la forme d'une bulle papale qui était ensuite acceptée par le roi de façon tacite ou explicite, ou celle d'un accord bilatéral (Concordat). Le Patronat a été la pierre de touche du modèle régalien des relations entre l'Église et l'État. En vertu de ce modèle, le roi catholique était à la tête de la structure politique de l'État. Il exerçait le pouvoir par prérogative divine et était donc considéré comme le vassal du pape. Les vice-rois, représentants du roi dans les colonies, avaient donc une double fonction : politique et ecclésiastique. Ils étaient chargés d'une part du gouvernement séculier, et d'autre part de l'exercice de ce que l'on appelle le droit de Patronat qui consistait à présenter des candidats aux postes ecclésiastiques, à décider de la fondation des Églises, de l'ouverture de monastères et de la création de nouveaux centres religieux. Les vice-rois étaient, dans les colonies, les plus hauts représentants de la religion catholique de la même façon que le roi dans

1. Il est certain que le point de départ de la discussion sur ces relations se situe au moment de l'invasion espagnole, puisque c'est à ce moment-là qu'apparaît la distance organique entre l'institution religieuse et l'État. La problématique sur les relations entre Église et État qui préoccupait déjà l'Europe est alors importée au "nouveau monde " à travers la conquête, et l'on ne peut pas véritablement parler de "relations Église État » avant cette date. Nous ne faisons pas seulement référence à l'inexistence évidente «d'Église » dans l'acception chrétienne du terme, mais à l'inexistence d'autonomie organique entre l'institution religieuse et l'État dans le monde précolombien qui ne connaît que le modèle moniste ou théocratique de gouvernement : l'Église est État et l'État est Église. 
la péninsule ibérique. Les prêtres de toutes catégories étaient fonctionnaires du vice-royaume et celui-ci se chargeait de lever la gabelle ecclésiastique, un impôt promulgué par la Bulle Donación. Celle-ci, octroyée par le pape aux rois catholiques, leur permettait d'exercer le droit de Patronat et de lever des impôts. Le revenu ecclésiastique était constitué par la dîme (10 \% des revenus de la terre) dont les deux/neuvièmes étaient destinés au ministère royal des finances. En outre, la couronne faisait payer les Bulles de la Sainte-Croix qui, au départ, servaient à financer les croisades et, par la suite, permirent l'obtention de faveurs ou d'indulgences. Les Églises paroissiales, créées sur l'emplacement de hameaux indigènes, étaient administrées par des prêtres qui rendaient compte au Conseil des Indes, organe politique du gouvernement dans le vice-royaume et aux Audiences. Tous ces éléments caractérisent l'étape du Patronat royal qui prend fin en 1821, date de l'indépendance péruvienne.

Une fois l'indépendance acquise, les indépendantistes, dans leur lutte pour la reconnaissance du nouveau régime, préfèrent ne pas multiplier les confrontations politiques et décident d'utiliser l'Église catholique comme courroie de transmission de leurs politiques et comme facteur de légitimation sur l'ensemble du territoire, comme les vice-rois l'avaient fait précédemment ${ }^{2}$. Ils n'ont donc pas modifié le type de relations existant depuis la colonie entre l'Église et l'État, et n'ont bien évidemment pas légiféré en faveur de la liberté religieuse, contrairement à ce qui s'est passé dans d'autres processus de libération politique du continent sud-américain. L'impact des processus révolutionnaires nord-américain et français et leur influence sur le pays se sont donc limités aux aspects idéologiques et politiques sans toucher le type de relations entre l'Église et l'État. Le Pérou n'a adopté de ces expériences de libération que ce qui permettait l'émancipation du colonialisme politique espagnol, sans y inclure la rupture avec le colonialisme religieux catholique romain.

Les gouvernants républicains ont, de fait et sans aucune coordination avec le siège du pouvoir central de l’Église catholique, assumé les prérogatives du Patronat royal comme un héritage historique, donnant ainsi naissance à un Patronat républicain de facto. Pour surmonter cette situation juridique irrégulière, conserver leurs attributions dans les affaires ecclésiastiques et bénéficier du soutien politique légitimateur du catholicisme, les responsables de l'indépendance du Pérou ont, dès le départ, cherché à régulariser intégralement les

2. Comme le vice-roi José de Abascal qui a demandé aux évêques résidant au Pérou d'utiliser leurs pupitres et leurs confessionnaux pour défendre l'ordre ancien, et dénoncer secrètement aux juges " tout suspect dont la conduite ou les conversations " démontreraient la filiation révolutionnaire. De la même façon, l'émissaire de Bolivar a demandé à l'évêque Goyeneche de « leur apprendre, par NSJ et à travers tous les ministres du culte, que le gouvernement de la république est juste et légitime, qu'ils doivent lui obéir, le servir et le défendre de toutes leurs forces, parce qu'il est l'œuvre de leurs mains et l'expression de leur volonté. » 
relations avec l'Église catholique en signant un Concordat entre le nouvel État péruvien et le Saint-Siège (Garaycoa, 1964).

Pour sa part, le Saint-Siège recevait les nominations de l'État péruvien aux places vacantes de l'épiscopat et émettait habituellement des bulles de nomination en faveur des candidats. Juridiquement, ces nominations ne reposaient pas sur les prérogatives supposées républicaines du Patronat mais bien sur les pouvoirs ecclésiastiques. C'est ainsi qu'en 1865, le secrétaire d'État du Vatican précise au gouvernement péruvien que le Saint-Siège ne reconnaît pas le droit de Patronat au président de la république et que ses communications n'ont donc valeur que de simples recommandations particulières (Pareja Paz Soldan, 1938 : 176). Il s'agissait donc d'un Patronat factice bien que le gouvernement péruvien l'ait toujours considéré comme une faculté constitutionnelle du président, l'inscrivant dans les divers textes des Constitutions politiques successives du Pérou.

Il faut noter qu'en 1874 le Saint-Siège reconnaît formellement ce "droit " au chef de l'État péruvien, à la suite d'une négociation assurée par l'envoyé du gouvernement à Rome, Pedro Gálvez. Il parvient à ce que le Pape envoie, le 3 mars 1874, des Lettres Apostoliques octroyant cette grâce à condition que les biens du clergé provenant de la dotation et de l'exercice du culte soient conservés et distribués fidèlement et que le gouvernement péruvien continue à favoriser et protéger la religion catholique comme unique culte reconnu et autorisé par l'État. De cette façon, l'État assure sa légitimité politique et la renforce à travers la légitimité religieuse, pendant que l'Église catholique consolide la sienne grâce au pouvoir coercitif de l'État.

En 1880, Nicolas de Piérola donne l'exequatur, l'approbation officielle des Lettres Apostoliques, mais le désir de régulariser les relations avec l'Église, grâce à un outil plus stable comme un Concordat, est inscrit successivement dans les Constitutions de 1828 à 1933. Cette dernière a été en vigueur jusqu'en 1979. De nombreuses tentatives de négociations pour signer un Concordat avec le Saint-Siège n'ont pas abouti pour différentes raisons et hasards de la politique (Garaycoa, $1965: 31$ ).

Si nous évoquons ici le problème du Concordat, c'est parce que, au Pérou, la nature juridique et politique des relations de l'État avec l'Église catholique a toujours influencé directement la politique envers les minorités religieuses et le degré de liberté religieuse autorisé. Lors de l'instauration de la République, il existait une relation étroite entre le catholicisme national, la religion d'État et l'intolérance religieuse. Par la suite, on observe un affaiblissement de la religion d'État avec une plus grande ouverture et plus de tolérance religieuse, en parallèle avec un catholicisme national toujours important qui tente de freiner le processus modernisateur et sécularisateur qui restera donc incomplet au Pérou. Il est paradoxal de constater qu'alors que la religion d'État se manifeste de plus en plus vigoureusement, il n'y a toujours pas de Concordat entre l'État et le Saint-Siège. 
Il faut attendre 1980 pour que celui-ci se concrétise alors que la laïcisation de l'État s'est déjà répandue dans de nombreux domaines de la sphère publique ${ }^{3}$.

Concernant les relations entre l'Église et l'État, le confessionnalisme doctrinal a été inscrit dans la Constitution pendant presque toute la période républicaine. Il est précisé que l'État professe la vraie religion, privilégiant une Église en particulier tout en excluant l'exercice public ou privé d'autres religions. L'étape constitutionnelle du confessionnalisme doctrinal est la continuation de la catholicité de l'État colonial inscrite dans les Constitutions républicaines et dont la dernière expression a été l'article 3 de la Constitution de 1839 : «sa religion [de l'État] est la religion catholique, apostolique, romaine, qu'il professe sans autoriser l'exercice public de tout autre culte. »

\section{Transformations des rapports privilégiés de l'État avec l'Église catholique}

Alors que divers aspects de la vie sociale sont touchés par la sécularisation et que certaines caractéristiques concrètes de l'ordre politique deviennent progressivement laïques (mariage civil, sécularisation des cimetières qui sont maintenant publics, abolition de la dîme et autres mesures du même type), une nouvelle étape constitutionnelle s'amorce. Les fondements constitutionnels qui donnent une position prépondérante à l'Église catholique se sécularisent d'euxmêmes, et leur légitimité n'est plus exclusivement religieuse mais elle se base sur des raisons de caractère public comme l'identité nationale. On voit alors se manifester clairement une idéologie nationale catholique qui associe l'appartenance nationale à l'appartenance religieuse. Celle-ci est considérée comme un facteur commun d'identité culturelle, fondement et unique bastion de l'unité politique d'un État incapable d'exercer une souveraineté territoriale complète et de réunir autour de lui les divers groupements sociaux qui se reconnaissent comme des nationalités. On pense ici aux peuples indigènes de l'Amazonie (Awajún, Achuar, etc.) qui, selon la Convention 169 relative aux Peuples indigènes, ont droit à «l'autodétermination» et dont les territoires sont reconnus comme «autonomes».

Il s'agit donc de l'invention d'un nouveau type de confessionnalisme d'État, à caractère sociologique et historique, qui commence de façon paradigmatique en 1915, lorsque la phrase « aucune autre religion n'est autorisée » est éliminée de l'article 4 de la Constitution. L'article est alors reformulé comme « la nation professe la religion catholique apostolique romaine et l'État la protège ». L’État ne se reconnaît donc plus comme catholique, mais prend note de l'appartenance

3. Je partage la définition de la laïcité proposée par Roberto Blancarte dans son article, dans ce numéro. Par ailleurs, je définis la sécularisation comme la "perte progressive de pertinence sociale et culturelle de la religión en tant que cadre normatif orientant les conduites et la vie morale de l'ensemble de la société " (Milot, 2008, p. 29). 
catholique de la population (fait objectif) et privilégie l'Église qui représente cette option religieuse en séparant la foi religieuse de la Nation de celle de l'État. Aujourd'hui, l'État favorise encore le catholicisme auquel adhère la majorité de la population.

L'explication immédiate de ce changement se trouve dans les graves problèmes d'intolérance religieuse, notamment contre les adventistes, qui surviennent dans le sud du pays. Le pouvoir économique des zones rurales, qui s'exprime à travers les intérêts des grands propriétaires, le pouvoir politique des caciques et des créoles, et le pouvoir du clergé, qui assure la légitimité de ces deux ordres de pouvoir, s'associent contre les paysans indigènes en une trilogie oppressive à la fin du XIXe et au début du XXe siècle. Cette hégémonie intégrale (politique, économique et religieuse) est alors menacée par l'entreprise de prosélytisme des adventistes (Église chrétienne d'origine nord-américaine) qui agit socialement par l'alphabétisation des indigènes, la promotion de la tempérance - pour contrer la consommation de boissons alcooliques et de substances hallucinogènes - et par une religiosité alternative de type protestant. La destruction par le feu d'une colonie indigène adventiste par des foules manipulées par un évêque catholique local dans le département de Puno, est à l'origine de la réforme constitutionnelle de 1915, lancée sous l'impulsion d'un député libéral.

Il faut ajouter à ce contexte le climat de confrontation entre libéraux et conservateurs dans toutes les discussions politiques de l'époque. Certains secteurs libéraux se radicalisent et forment des groupes spéciaux qui sont liés à des courants philosophiques athées et francs-maçons. On voit alors apparaître l'anticléricalisme militant de certains penseurs comme Manuel Gonzales Prada, Alberto Quimper, Abelardo Gamarra et Pablo Pátron qui fonderont le Parti Union Nationale dont le programme entend laïciser l'État en établissant la liberté de culte, le mariage et les cimetières civils et l'école neutre.

La nécessité de tolérer les divers cultes religieux pour stimuler la modernisation de l'économie à travers la migration étrangère (principalement européenne) est la première revendication de l'État laïque péruvien. Mais cela ne s'arrête pas là. Le libéralisme progressiste ouvre un débat sur la sécularisation des cimetières catholiques et sur le mariage civil. Suite à plusieurs problèmes de discrimination entourant la sépulture de célèbres personnages anticléricaux qui soulevèrent l'indignation de l'opinion publique, le gouvernement crée des cimetières civils. Il légifère également sur la validité de l'inscription du mariage civil pour les étrangers non catholiques qui épousent des catholiques péruviens, en évitant que la loi ne se basent sur des dogmes catholiques et que ces personnes ne soient traitées comme des concubins au lieu d'époux légitimes.

Après 1915, la laïcisation de l'État progresse - non sans mal - dans une période qui se caractérise par une extension des conquêtes légales en faveur de la liberté religieuse et de nombreuses pressions pour obtenir une plus grande séparation entre l'Église et l'État. Avec l'historien Asín Armas (Armas, 2000 : 111), 
nous pouvons distinguer trois étapes fondamentales de ce processus. La première, de 1920 à 1940, est une période marquée par la présence de régimes politiques de type régalien - et en ce sens, ils prolongent l'esprit colonial des relations entre l'Église et l'État - mais qui, comme nous l'avons déjà dit, se laïcisent progressivement. À cette époque, les présidents Augusto Leguía, Luis Sanchez Cerro, Oscar Benavides et Manuel Prado maintiennent, chacun à sa manière, avec des hauts et des bas, de bonnes relations avec l'Église catholique qu'ils défendent depuis leur position nationale catholique. On observe aussi, durant cette période, des progrès et des reculs concernant la tolérance religieuse récemment inscrite dans la Constitution.

Dans ce contexte, de plus en plus pluraliste, l'Église catholique cherche une alliance solide avec le Gouvernement du Président Augusto Leguía au moyen de la consécration du Pérou au «Sacré Cœur de Jésus », en mai 1923. Ceci provoque de grands mouvements de protestations, instigués par des ouvriers, des étudiants et des intellectuels et conduits par le jeune Víctor Raúl Haya de la Torre (futur fondateur de l'Alliance Populaire Révolutionnaire Américain, APRA, parti social-démocrate historique et très important au Pérou). Ce leader, porté par des influences protestantes et maçonniques, est en quelque sorte le "père » symbolique de la future Constitution du 1979. La déclaration de principes de ce mouvement a été importante en proposant l'instauration d'un État laïque en Pérou:

« De protester publiquement contre la consécration de la République au culte du Cour de Jésus.

De demander la séparation absolue entre l'Église et l'État.

De faire la reforme du Article 5 de la Constitution afin de reconnaître explicitement la liberté de culte et de déclarer que l'État ne professe ni ne protège aucune religion.

De délaisser l'actuel enseignement dogmatique au moyen des lois en supprimant les pratiques et cours religieux obligatoires, en les remplaçant par des principes moraux qui peuvent instruire par la raison, la tolérance et l'idéal.

De demander l'inclusion dans le nouveau Code Civil de l'obligation du mariage civil sans aucun acte religieux, l'institution du divorce approuvée déjà par le Congrès, et la suppression des tribunaux ecclésiastiques qui sont une atteinte à la souveraineté nationale.

De supprimer la section première du livre 2 de notre archaïque Code Criminel sur les infractions contre la religion catholique ${ }^{4}$.

Le résultat est l'annulation de la cérémonie politico-religieuse de consécration du Pérou. En 1930, l'Archevêque responsable de cette alliance avec le Président Leguía, Mgr Emilio Lissón, est forcé de présenter sa démission ; puis survient le coup d'État qui renverse le Président.

Cependant, le protestantisme s'était déjà développé, même en l'absence d'un régime légal de tolérance religieuse, et les premières manifestations de la récente diversité religieuse institutionnelle apparaissent au Pérou. En 1898, s’implante

4. Publié dans le journal El Tiempo, le 24 mai 1923, p. 3. 
l’Église évangélique méthodiste et la Société biblique américaine, puis l’Église adventiste du Septième Jour. En 1903, l'Union évangélique sud-américaine et l'Église de la Sainteté s'implantent; en 1908, c'est au tour des Assemblées de Dieu; ensuite, en 1910, l’Armée du Salut s'installe au Pérou, suivie, en 1914, de l'Église du Nazaréen et de la Société biblique britannique; puis, en 1916, l'Église libre d'Écosse et, en 1925, l'Église baptiste s'y établissent également.

La deuxième période qui va de 1940 à 1970, signifie la «nationalisation » sociale des expressions religieuses non catholiques étrangères, l'expansion des cultes évangéliques et, de ce fait, un certain nombre de débats et conflits publics entre les leaders protestants et le clergé catholique. Ces conflits conduisent à l'adoption, en 1945, d'une loi interdisant les cultes publics non catholiques, mais cette loi est rapidement abolie grâce, notamment, aux recours juridiques des avocats évangéliques. Enfin, la troisième période, qui va de 1970 à 1990, est caractérisée pour l'explosion de la pluralité religieuse et la participation des évangéliques dans la politique nationale.

La population du Pérou (en 2008), d'environ vingt-neuf millions deux cent mille personnes, compte une majorité de catholiques ( $89 \%$ ). Mais les dénominations protestantes et les autres religions ont augmenté de $140 \%$ entre les années 1990 et 2002. Ainsi, les missions évangéliques, les Adventistes du Septième Jour, les Témoins de Jéhovah et les autres confessions représentent maintenant $11 \%$ de la population péruvienne.

\section{La lente évolution des libertés religieuses des minorités}

En superposant le développement constitutionnel des relations entre l’Église et l'État et la liberté religieuse, on peut faire le lien entre les constitutions empreintes de confessionnalisme et la reconnaissance progressive de la liberté religieuse comme un droit et non plus comme une simple tolérance des cultes. Par exemple, la constitution de 1920 reconnaît, dans son article 5, que la Nation professe le catholicisme et que l'État le protège. Toutefois, il est formulé à l'article 23 que personne ne peut être poursuivi pour ses croyances (ce qui est une formule négative de la liberté religieuse). Dans la constitution de 1933, on affirme en même temps que l'État protège le catholicisme à cause des sentiments religieux de la majorité nationale, et que les autres cultes jouissent de la liberté. Puis, dans la constitution de 1979 , l'article $2(\$ 3)$ comporte une forme de reconnaissance (positive et non plus négative) de la liberté religieuse individuelle et collective. Cependant, l'Église catholique est encore l'objet d'un traitement spécial, par le biais de la reconnaissance constitutionnelle de sa personnalité juridique et de son rôle dans l'histoire nationale (Pareja Paz Soldán, 1938 : 175-176).

Ce sont les constitutions de 1979 et 1993 qui formulent le plus clairement un nouveau type de relation entre l'État et les minorités religieuses. En plus de reconnaître la liberté religieuse des citoyens et des institutions religieuses non 
catholiques, elles indiquent que l'État peut, d'une certaine façon, collaborer avec elles. Les textes constitutionnels péruviens vont ainsi graduellement parvenir à la tolérance des cultes non catholiques, puis reconnaitre la liberté de culte et la non-persécution des croyances. Finalement, au-delà de la liberté de culte et la garantie de la liberté religieuse, un statut sera accordé aux collectivités religieuses non catholiques et à leurs membres. Avec l'accroissement du pluralisme religieux et le rôle plus accentué, sur la scène politique nationale, principalement des évangélistes, ce statut constitutionnel apparaît trop général et peu fonctionnel aux yeux des minorités religieuses qui ont de nouvelles aspirations et de nouvelles nécessités. Elles vont donc, très rapidement, revendiquer non plus seulement la liberté religieuse mais son élargissement en exigeant l'égalité religieuse.

Ces aspirations sont forgées par les ambiguités d'une société qui semble culturellement tolérante à l'égard de la diversité religieuse, à condition de ne rien perdre des privilèges reconnus à l'Église catholique. En fait, les normes légales continuent de suivre la logique du Patronat et de la religion officielle. On constate, par exemple, que la reconnaissance générale de la liberté religieuse inscrite dans la Constitution est insuffisante, et inefficace, dans les situations où les libertés religieuses individuelles des membres des minorités religieuses sont compromises (Huaco, 2005).

En étudiant divers cas juridiques où la diversité religieuse a été l'objet d'un jugement, il est évident que, de manière formelle ou non, prédomine toujours la conscience de vivre dans un pays catholique qui tolère les manifestations religieuses des minorités et accepte de cohabiter pacifiquement et harmonieusement avec elles à condition qu'elles ne portent aucune atteinte à ce qui est considéré comme la "normalité » ou les normes communes. C'est le cas, par exemple, d'étudiants universitaires adventistes qui ont porté leur cause devant l'Ombudsman à propos de conflits d'horaires entre les obligations académiques et le jour du culte, le samedi ${ }^{5}$. Les universités péruviennes considéraient qu'elles respectaient la liberté de croyance religieuse mais qu'elles n'avaient pas pour mandat de concilier les obligations académiques avec les pratiques religieuses. Elles ont alors refusé toute possibilité de trouver des " accommodements raisonnables » afin de permettre aux étudiants de respecter leurs pratiques religieuses et leurs obligations académiques. Selon l'Ombudsman, la liberté religieuse doit être respectée dans les établissements universitaires et le Tribunal Constitutionnel - sur un cas similaire survenu au sein du pouvoir judiciaire - a même affirmé que "ce serait une absurdité de permettre le développement des croyances religieuses mais non leur pratique ${ }^{6}$. L'État et les institutions publiques mettent donc en œuvre une certaine tolérance religieuse mais n'assurent pas toujours toutes les conséquences

5. Les cas cités ont été défendus par l'auteur et correspondent aux Communications d'Ombudsman No. 073-2005-DP/AAC, 1026-2006-OD-LIMA/DESC, 07-2006-DP/AAC et 1252-2008-DP-OD-CALLAO.

6. Dans le cas No. 895-2001-AA/TC. 
juridiques de la "liberté » religieuse inscrite dans la Constitution. En outre, il faut dire qu'il n'existe pas vraiment une culture des droits de l'homme au sein de la société, de nombreux citoyens ayant une connaissance assez faible de ceuxci et des recours possibles.

\section{La position juridique privilégiée de l'Église catholique}

On voit poindre, toutefois, une prise de conscience de la place que devraient occuper les Églises minoritaires dans la société, ce qui donne lieu, parfois, à une critique directe contre le caractère quasi-concordataire entre l'État et l'Église catholique, qui paraît même s'être attribué le rôle de pierre de touche d'une discrimination religieuse systématisée au sein des instances du pouvoir. Ainsi, on constate la situation concrète de discrimination sociale envers les minorités religieuses d'une part et, d'autre part, on observe que l'ordre juridique et l'État assurent un traitement préférentiel à l'Église catholique à la suite de l'accord signé, en 1980, entre le Saint-Siège et le Pérou et qui représente un changement fondamental dans le régime de relations entre l'Église et l'État. Cette réforme des relations entre l'Église et l'État fait suite à une initiative de l'Église catholique ${ }^{7}$ approuvée par la conférence épiscopale péruvienne qui affirme: " tous sont d'accord (sic) pour dire qu'il faut demander à l'État péruvien de renoncer au droit de Patronat... qui est anachronique et mal adapté (sic) à la mentalité actuelle... ». Le Concile ayant défini les relations idéales entre l'Église et l'État comme des relations "d'autonomie et d'indépendance ", mais avec "coopération ", il faut dépasser le régime régalien d'interventionnisme de l'État sur l'Église, représenté par le Patronat et ses caractéristiques : bulles, exequatur, nominations des dignitaires ecclésiastiques, etc. Le document El Constitucionalismo peruano y sus problemas affirme que le statut ecclésial particulier de l'Église catholique devrait se traduire par l'introduction dans le texte constitutionnel d'une mention spéciale sur la relation privilégiée entre l'Église et l'État (Bernales et Rubio, 1998).

Les négociations entre l'Église catholique et l'État ont eu lieu pendant trois jours très mouvementés et, le 19 juillet 1980, a été signé l'accord entre le SaintSiège et la République du Pérou. Il fut ratifié, le 22 juillet 1980, par le Pape JeanPaul II, et par le gouvernement du Pérou deux jours plus tard, sans médiation du Congrès contrairement à ce qui est stipulé par la Constitution de 1933 alors en vigueur ${ }^{8}$. Il faut dire que cet accord a été signé par un régime dictatorial qui, logiquement, n'a pas de pouvoir législatif indépendant. La norme légale d'approbation de cet accord a été publiée, l'après-midi du vendredi 25 juillet,

7. Comme le prouve le document intitulé : El Constitucionalismo peruano y sus problemas. Selección de textos para el Primer Curso de Derecho Constitucional Peruano. García Belaúnde, Domingo. PUCP, Programme universitaire de droit, Lima, 1970.

8. Article 154 - Sont attributions du président de la République : «...22) signer des Concordats avec le Saint-Siège, en suivant les instructions données par le Congrès » 
dans une parution inhabituelle de seulement deux cents exemplaires du Journal Officiel. Le changement des instruments de ratifications fut confirmé le samedi 26 juillet 1980, deux jours avant l'entrée en vigueur de la nouvelle Constitution politique approuvée en 1979 (qui n'oblige plus à signer des concordats avec le Saint-Siège et exige que tout traité international soit soumis à l'approbation préliminaire du Congrès avant d'être présenté au Président de la République). Cet accord s'inscrit dans le contexte plus large de la doctrine du concile Vatican II sur les relations entre l'Église et les États et sur la liberté religieuse. Ce concile s'est prononcé en faveur de la liberté religieuse comme droit inhérent à la personne humaine, mais il a plaidé à faveur d'une version atténuée de l'État confessionnel $^{9}$. Il est important de noter que si pendant l'époque préconciliaire les questions de liberté religieuse et confessionnalisme de l'État sont intimement liés, lors du concile les deux thèmes vont être séparés. La raison en est évidente : il faut reconnaître et se prononcer clairement en faveur de la liberté de culte sans renoncer à ce que l'État établisse des régimes spéciaux en faveur de l'Église (De Fuenmayor, $1974: 123$ ssq.) ${ }^{10}$.

Grâce à ce Concordat, la source de production normative qui régule les relations entre l'État et l'Église catholique est le produit d'un traité international avec le Saint-Siège, signé en 1980, alors que pour réguler le statut juridique des minorités religieuses, il existe une législation d'État unilatérale. Il en découle diverses formes d'inégalités dans la façon dont l'État traite les religions institutionnalisées. Les droits reconnus à l'Église catholique sont inscrits dans un instrument de droit international alors que ceux accordés aux minorités dépendent du droit interne. On reconnaît pleinement les formes d'organisation propres à l'Église catholique, mais les minorités religieuses doivent s'organiser comme associations civiles sans but lucratif. On reconnaît la personnalité juridique civile et la capacité légale de l’Église catholique de deux manières : par Concordat et par voie constitutionnelle, alors que les minorités sont soumises à une inscription dans un registre de niveau juridique inférieur. Le financement de l'État est direct (assignation, subvention, donation) et indirect (bénéfices fiscaux, exonérations et désaffectations) pour le catholicisme, mais seulement indirect et à un niveau inférieur pour les minorités religieuses. Il existe une normativité exclusive pour l’Église catholique et une autre pour les minorités religieuses.

9. « $\mathrm{Si}$, tenant compte des conditions particulières des peuples, on donne à une communauté religieuse une reconnaissance civile particulière dans l'ordre juridique de la société, il est nécessaire que la liberté en matière religieuse soit reconnue et respectée pour tous les citoyens et communautés religieuses. " (Concile Vatican II, 1998: 423)

10. «Il faut dire aussi que le Concile Vatican II n'a pas choisi les doctrines de certains penseurs catholiques qui estimaient que la défense de la liberté religieuse était incompatible avec un État confessionnel catholique. Le Concile a surmonté cette contradiction apparente et a établi la compatibilité d'un régime de liberté religieuse avec le confessionnalisme catholique ou non catholique de l'État. " 


\section{Les aspirations politiques des élites évangéliques}

Les élites évangéliques réagissent de différentes façons face à cette relation privilégiée entre l'État et l'Église catholique. Certains perçoivent ce système simplement comme le fait d'un pays historiquement catholique ayant encore des réminiscences du système régalien et qui peut rester ainsi à condition que les droits et les avantages des minorités se voient améliorés. Pour d'autres, il est vécu comme un obstacle inacceptable dans un régime de totale égalité religieuse et qui devrait absolument disparaître (même si, de façon paradoxale, ils ne sont pas contre l'idée de bénéficier d'un statut similaire).

Cette différence d'attitude face à l'influence politique et à la position hégémonique du catholicisme sur l'État s'exacerbe lors du débat entourant la rédaction de l'article 71 du projet de nouvelle Constitution (2002) sur les relations entre l'Église et l'État, et provoque l'institutionnalisation de la division de fait du camp évangélique et la naissance de l'Union des Églises chrétiennes évangéliques du Pérou (UNICEP). La polémique constitutionnelle n'est pas directement la cause de cette division institutionnelle, mais elle contribue, sans doute, à faire éclater au grand jour les controverses antérieures. Bien évidemment, cette divergence s'alimente d'autres désaccords concernant la manière d'intervenir dans la politique nationale : soit depuis les autorités religieuses proches du fondamentalisme, soit à partir de positions chrétiennes laïques. Tout cela semble paradoxal puisque lors du débat constitutionnel de 2002, l'élite évangéliste du Concile national évangélique (CONEP), favorable au point de vue laïque et non confessionnel de la relation entre politique et religion, selon ses leaders et ses discours publiques, a soutenu les prétentions de l'épiscopat visant à maintenir dans la Constitution une reconnaissance juridique favorisant l'Église catholique. Cette élite évangéliste considérait cette reconnaissance comme une valeur purement historique et symbolique, liée à l'histoire péruvienne et non pas comme à une norme juridique. Peut-être que la proximité du CONEP et de l'épiscopat au sein d'un organisme œcuménique, le Comité interconfessionnel, a joué un rôle clé dans cet accord. Pour leur part, les élites religieuses plus radicales dans leurs conceptions politicoreligieuses se sont déclarées favorables à "l'égalité religieuse » et opposées au confessionnalisme catholique de l'État.

Ce jeu entre les forces religieuses et politiques est influencé par les modes de participation politique des groupes religieux. On peut voir que, dans un premier moment historique (début du $\mathrm{XX}^{\mathrm{e}}$ siècle), les protestants sont visibles dans les mouvements sociaux et intellectuels comme la lutte pour les huit heures de travail maximum par jour, l'indigénisme, la liberté religieuse et contre l'alcool et le tabac. Les autres champs d'intervention politique des évangélistes sont l'intégration aux partis politiques, avec volonté de devenir fonctionnaires dans les organismes publics étatiques ou parlementaires, soit en tant que candidats individuels, soit en constituant des partis politiques confessionnels. C'est ainsi que, dès la décade de 1970, il y a des membres du clergé évangélique qui tentent d'accéder au 
Parlement dans le cadre du parti politique APRA et sous l'idéologie politique de ce parti. Deux d'entre eux réussissent : José Ferreira (1962 et 1985) et Pedro Arana (1978). Dans les années quatre-vingts, l'action politique se fait plus ambivalente. On voit émerger de nouveaux partis politiques évangéliques où le critère d'adhésion est religieux mais aussi collectif (il s'agit d'une dénomination exclusivement). D'autres évangélistes s'intègrent au sein de partis politiques séculiers, à titre individuel. Un membre du clergé pentecôtiste, Humberto Lay, se présentera même aux présidentielles de 2006 à la tête de son parti indépendant et confessionnel, la «Restauration Nationale».

Aujourd'hui, on observe une tendance croissante à la «colonisation » de la sphère publique et ce par le moyen d'alliances entre des évangélistes fondamentalistes et des « intégristes » catholiques. L'intention qui réunit ces deux camps est d'influencer l'État sur des questions de politiques publiques, notamment la sexualité, l'avortement, la reproduction et la contraception, politiques encore largement déterminées par la morale chrétienne. Il y a ainsi un risque d'instrumentalisation des débats concernant la liberté et l'égalité religieuses afin d'affermir la légitimité des divers mouvements religieux et des Églises et ainsi, de favoriser une plus grande participation politique des croyants afin d'augmenter la possibilité d'influencer les lois, les politiques publiques et les valeurs de l’État dans le sens de la morale chrétienne.

\section{Le principe constitutionnel d'égalité en matière de religion}

La question de l'égalité religieuse et la mention de l'Église catholique dans la Constitution ont été l'objet de fortes préoccupations dans le débat constitutionnel récent. Celui-ci avait été précédé d'une interrogation, de la part de certains congressistes, sur les versements mensuels directs reçus par les membres du personnel ecclésiastique catholique. L'avant-projet de la commission de réforme constitutionnelle du Congrès de la République reproduit l'option de l'article 50 de la Constitution de 1993 qui signale que l'État "prête » sa collaboration à l'Église catholique alors qu'avec les autres confessions, il «peut établir » une collaboration. Cette formulation est modifiée avant le débat au Parlement, alors que les différentes positions qui vont s'affronter commencent à se dessiner. La conférence épiscopale propose de maintenir le texte de l'article 50 de la Constitution en vigueur sans le modifier. Le Concile national évangélique propose un changement ne mentionnant pas l'Église catholique et insiste sur l'idéal d'égalité religieuse. Mais, d'un autre côté, un groupe de parlementaires soutenus par des secteurs religieux divergents du CONEP présentent le projet No 3156, intitulé "Respecter le principe d'égalité religieuse ", qui élimine la mention au catholicisme, l'engagement de coopération de l'État avec toutes les religions et qui ratifie explicitement l'égalité religieuse. Ceci démontre publiquement les controverses soulevées par cette initiative et les dissensions avec le 
Comité interconfessionnel (collectif interreligieux, partisan de la mention explicite de l'Église catholique conformé par la conférence épiscopale catholique, l'Église anglicane, l'Église catholique orthodoxe, l'Église luthérienne, l'Église méthodiste et la communauté juive).

Le débat parlementaire (2002) sur l'article 71 concernant les relations entre l'Église et l'État débute dans un climat surchauffé. Le camp non catholique est divisé entre le Comité interconfessionnel et les différents groupes religieux; des éditoriaux et articles de presse annonçent une attaque athée et anticléricale cherchant à instaurer un État laïque antireligieux ${ }^{11}$, et un rapport du Défenseur du Peuple (Ombudsman) soutient juridiquement l'élimination de la mention de l'Église catholique dans la Constitution. L'article, finalement approuvé, ignore la position des divers groupes non catholiques opposés à la fameuse mention : l'Église adventiste du Septième Jour, la communauté bahaie, les mormons, les israélites du Nouveau Pacte universel, la communauté islamique, notamment, et quelques Églises évangéliques dissidentes du CONEP (des églises néo-pentecôtistes entretenant des rapports étroits avec le fondamentalisme évangélique nord-américain qui, ensuite, vont créer l'UNICEP). L'article se lit comme suit :

Article 71: «Dans un souci d'indépendance et d'autonomie, l'État reconnaît l'Église catholique comme un élément important de la formation historique, culturelle et morale du Pérou et lui prête sa collaboration.

L'État reconnait et respecte toutes les confessions religieuses et établit des accords de collaboration avec elles à travers leurs organes représentatifs, selon un critère d'équité. »

Parallèlement à la situation précaire des libertés religieuses individuelles et collectives que nous avons décrites à grands traits, on assiste, depuis 1990, à une irruption notoire de la participation politique des évangélistes. Les candidatures politiques de pasteurs évangéliques et adventistes se multiplient et l'on assiste à des essais de création de partis politiques confessionnaux. Les débats sur la « liberté et égalité religieuse » organisés par diverses confessions se multiplient sous la direction de leaders individuels issus des diverses Églises évangéliques qui perçoivent le potentiel politique et électoral que représente la bannière de l'égalité et de la liberté religieuse pour obtenir un siège au Congrès de la République. Tous ces éléments démontrent que le thème de l'égalité religieuse va se convertir en un débat public - au moins dans les cercles ecclésiastiques non catholiques - qui, dépassant ce domaine, prend place dans le débat national et passe d'une revendication marginale et isolée à une défense collective et politique. On ne revendiquera plus la liberté de culte ou la liberté religieuse, on ne parlera plus d'État laïque, mais d'égalité religieuse complète.

Actuellement, c'est précisément dans l'espace public que se reflète l'importance croissante du thème des relations entre l'État et les minorités religieuses.

11. Vidéo et article "Dieu et César " du congressiste conservateur et membre de l'Opus Dei, Rafael Rey, paru dans El Peruano, mardi 25 mars 2003. 
Plusieurs projets de loi proposés par les partis politiques non confessionnels se succèdent pour canaliser politiquement le marché électoral non catholique et tenter de construire un nouveau cadre juridique. Le premier d'entre eux, le «projet de loi de liberté religieuse pour le Pérou », élaboré en 1998, est présenté comme projet de loi No 894, le 3 octobre 2001. Il s'agit du premier projet de loi officiel sur la liberté religieuse de toute l'histoire parlementaire péruvienne. Ce projet - comme tous ceux qui ont été présentés jusqu'à aujourd'hui - ne reçoit pas l'appui de l'Église catholique. Au contraire, elle s'y oppose bien que l'article $11^{12}$ propose que la future loi de liberté religieuse soit applicable seulement de manière accessoire à l'Église catholique, à cause de l'accord de Concordat que le Saint-Siège a signé avec l'État péruvien en 1980. Ce projet n'a pu être soutenu par les minorités non catholiques car elles n'ont jamais été consultées sur son contenu.

\section{La régulation institutionnelle d'une laïcité encore partielle}

Les facteurs contextuels qui sont politiquement à l'origine des divers projets de loi tentant de réguler le phénomène religieux ont également provoqué l'apparition d'un nouveau cadre légal. Le premier pas important dans la régulation formelle et juridique des relations de l'État avec les minorités religieuses est la création de la Direction des problèmes inter-confessionnaux, organe directeur de la Direction nationale de la Justice, grâce au décret suprême (DS) No 0262002-JUS qui modifie le règlement de l'organisation et des fonctions du ministère de la Justice ${ }^{13}$. Parmi les considérations de ce décret suprême, il faut évoquer l'article 2 de la Constitution sur le droit à la liberté religieuse et à la liberté de conscience, l'article 50 sur les relations de coopérations de l'État avec les confessions religieuses, et mentionner que, selon l'article 5 de la loi organique du secteur justice (Loi No 25993), ce ministère est chargé d'assurer la coordination de la relation entre le pouvoir exécutif et l'Église catholique et les autres confessions. Pour conclure, il est signalé que « par conséquent, il faut adapter la structure organique du Ministère de la Justice pour y implanter les mécanismes permettant de faire la promotion des relations de l'État avec d'autres confessions ".

Les fonctions de la nouvelle Direction vont donc renforcer celles qui existent déjà à la Direction des affaires de l'Église catholique avec certaines différences concernant la mise en place de juridictions ecclésiastiques et la légalisation des actes des autorités ecclésiastiques catholiques qui ne concernent que la première. Cette nouvelle norme prévoit que la nouvelle Direction doit « diriger et coordonner des actions qui améliorent les relations du pouvoir exécutif et du Secteur avec

12. «Art. 11 - Les relations entre l'État péruvien et l'Église catholique sont régies, en ce qui concerne la situation juridique de celle-ci et la collaboration entre les deux, par les dispositions de l'accord entre le Saint-Siège et la république du Pérou, approuvé par le décret de loi N 23211 ».

13. DS N 026-2002-JUS, (26-VII-02). Normes légales, tome 324, juillet 2002, p. 64. 
d'autres confessions non catholiques pour, quand l'État l'exige, renforcer la liberté religieuse".

Le décret suprême (DS) No 003-2003-JUS est une seconde norme légale très importante qui prévoit « la modification du décret sur le processus des donations destinées à l'Église catholique et instaure le registre des religions non catholiques. » Le décret suprême No 025-93-JUS est donc modifié en fonction de cette norme : les dons reçus par l'Église catholique et les autres religions seront gérés par la Direction nationale de Justice, à travers les directions existantes. Il est prévu d'instaurer un registre qui permettra à la nouvelle Direction des affaires interconfessionnelles de remplir ses fonctions et assurera le développement des procédures administratives. Le DS No 003-2003-JUS (au-delà de son contenu qui semble contestable dans certains aspects, par exemple en différenciant les droits de l'Église catholique et ceux des groupes non catholiques ou en définissant ces derniers par opposition au catholicisme) est un évènement historique pour le droit des religions au Pérou, puisque c'est la première fois de l'histoire qu'un tel registre est créé.

Le second article de ce DS No 003-2003-JUS décrète que la nouvelle Direction des affaires interconfessionnelles est responsable de la mise en place du "Registre des confessions non catholiques » pour répondre aux spécifications du DS No 026-2002-JUS c'est-à-dire : « le renforcement de la liberté religieuse » et la promotion des relations entre l'État et les confessions non catholiques. On peut également y lire que : "seront considérées confessions non catholiques les personnes juridiques inscrites dans les registres publics. Les autres obligations et formalités nécessaires à l'inscription des confessions, et dont il est fait référence dans cet article, seront approuvées par résolution ministérielle». Le législateur péruvien a donc décidé de continuer à obliger les religions minoritaires à s'organiser comme des «associations civiles à but non lucratif ", au lieu de respecter leur identité organisationnelle. Il décide tacitement que les nouvelles confessions religieuses devront se constituer sous le régime d'association pour être inscrites dans le nouveau registre des confessions «différentes de la catholique ».

Pour respecter l'article 2 du décret suprême, le ministre de la Justice a recueilli des opinions et des suggestions à travers la résolution ministérielle No 020-2003JUS, puis a décidé de la pré-publication d'un projet de « règlement du registre des confessions religieuses différentes de la catholique ». Le résultat de ces enquêtes a permis l'élaboration et la publication d'un nouveau projet : " normes applicables au registre des confessions religieuses différentes de la catholique ", à travers la résolution ministérielle No 337-2003-JUS. Ce nouveau régime légal a permis l'inscription de groupements religieux non catholiques au nouveau registre instauré ${ }^{14}$. Un comité assesseur en affaires interconfessionnelles a été créé au sein du ministère de la Justice, formé par des représentants des groupes religieux.

14. Dès ces années jusqu'à aujourd'hui, il y a cent vingt-cinq groupes religieux inscrits. Voir : http://www.minjus.gob.pe/registro_de_confesiones.html (consulté le 30 mars 2009). 
L’État a lui-même convoqué les futurs membres du comité assesseur (une instance publique par essence) en fonction de leur représentativité religieuse, convertissant ce groupe en une instance de coordination inter-ecclésiastique, alors qu'il aurait dû être constitué d'un groupe de fonctionnaires publics laïques, aidant l'État à formuler une politique religieuse en fonction d'intérêts plus généraux. Cette situation s'explique par le fait qu'il s'agit d'un premier essai et aussi parce que les fonctionnaires, qui ne connaissent que les relations institutionnelles avec l'Église catholique, comprennent mal les principes et les critères qui régissent la politique religieuse de l'État en matière d'égalité. De fait, la convocation du ministère de la Justice visant à former des corps consultatifs donnant leur opinion sur la nouvelle loi de liberté religieuse était davantage basée sur une conception de l'État pluriconfessionnel que sur celle d'un État laïque.

Cette normativité qui sera probablement l'objet d'une révision législative à court ou moyen terme, à cause de ses multiples failles, constitue néanmoins un grand progrès dans la formalisation des relations institutionnelles des confessions avec l'État. En 2005, elle a permis l'instauration d'un groupe de travail interconfessionnel, convoqué par le ministère de la Justice à travers la résolution ministérielle No 070-2005-JUS, qui est à l'origine du premier « projet de loi sur la liberté et l'égalité religieuse ", élaboré avec la participation des mêmes minorités religieuses auxquelles la norme finalement approuvée est destinée. Ironiquement, même si ce projet a été fait sous sa direction, il n'a pas été apprécié par le ministère de la Justice ni par l'Église catholique et celle-ci lui opposa son veto. Cette difficulté a été récemment surmontée à la demande de la cellule parlementaire Aprista (parti de gouvernement) qui a présenté le projet de loi No 1008/ 2006-CR « sur la liberté et l'égalité religieuse » qui reprend totalement le projet du groupe de travail interconfessionnel. Ce projet est le dernier d'une longue série de tentatives pour créer un cadre légal définitif qui formalise les relations entre l'État et les minorités religieuses au Pérou.

En plus des formidables innovations en matière de droit individuel et collectif de ce projet, on note une certaine aspiration à partager avec l'Église catholique la position de groupe privilégié et la mentalité patrimoniale que nous avons présentées, par exemple lorsqu'on inclut des articles concernant les impôts et l'établissement d'accords avec l'État pour élargir les domaines de coopération de l'État avec les groupes religieux.

Cette initiative de loi est en accord avec ce qui a été approuvé dans le projet de Constitution de 2002, (sur le critère d'équité). Il s'agit d'introduire au Pérou le système d'accords ou de "législation pactée " en suivant l'exemple de l'Espagne, et d'autres pays comme l'Italie et l'Allemagne, où seulement quelques confessions non catholiques établissent des accords avec l'État pour régler des problèmes d'intérêt commun. Ces accords ont un caractère de droit interne et sont donc de rang inférieur à ceux signés avec le Saint-Siège (qui sont des traités internationaux). Les contenus thématiques de ces accords concernent la tutelle 
du droit de liberté religieuse sur les fêtes religieuses et les jours fériés, le statut des ministres du culte, la reconnaissance civile du mariage religieux, l'assistance religieuse dans les hôpitaux, les casernes et les prisons, le régime alimentaire. Mais ces accords se rapportent aussi au régime immobilier des lieux de culte, à la protection du patrimoine historique, culturel et artistique, aux produits et aux actes rituels, au service militaire alternatif, à l'enseignement religieux et au régime fiscal particulier et l'accès aux moyens de communication (Fernandez Coronado, 1995). Tous ces aspects des législations à propos de la religion pourraient être régis par une loi d'État et non par un accord dont la nature même augmente le risque d'inégalités concernant la liberté religieuse.

Les derniers évènements survenus au Pérou - lors de la discussion sur la légalité de "la pilule du lendemain", de l'avortement thérapeutique et de l'approbation définitive de la convention ibéro-américaine des droits des jeunes, dans laquelle pointe la reconnaissance du droit au mariage entre personnes du même sexe - confirment une identification croissante et une alliance entre les secteurs religieux évangéliques et catholiques autour d'un agenda politique commun. Celui-ci s'oppose aux mouvements civils en faveur des droits sexuels et reproductifs, ce qui, selon notre analyse, accentue la tendance qui voudrait que l'État péruvien passe d'État monoconfessionnel à État pluriconfessionnel au lieu de devenir un État laïque.

\section{Entre laïcité et pluriconfessionnalisme}

L'État péruvien semble répondre aux exigences de sécularisation et de laïcité selon une double stratégie. En ce qui concerne les relations entre les Églises et l'État, il paraît accorder le même traitement légal aux minorités religieuses (qui considèrent que l'égalité religieuse est synonyme d'État laïque) pour les rapprocher du statut de religion officielle dont jouit encore l'Église catholique. En outre, il estime que, politiquement, il lui convient de ne pas respecter le Concordat qui le lie au Saint-Siège. En ce qui concerne les politiques publiques sur la sexualité, la reproduction, la santé et l'éducation, il essaie de prendre ses distances avec les directives morales et les dogmes catholiques, en sachant que cette distance ne sera pas rejetée politiquement par la population même si celle-ci est majoritairement catholique.

La classe politique péruvienne, y compris le parti au pouvoir, semble considérer qu'un État lä̈que correspond davantage à un pluri-confessionnalisme interreligieux, avec une Église dominante, fortifiant le confessionnalisme de l'État mais dans un système pluriel. L'Église catholique favorise aussi cette orientation, puisqu'elle se manifeste en faveur d'une loi qui accorde un statut partiellement égalitaire aux minorités religieuses, sans que cette égalité soit totale (conservation de privilèges) et à condition que l'accord avec le Saint-Siège soit toujours respecté. 
C'est une façon d'agir pour éviter que la société péruvienne ne se dirige vers des formes de gouvernement et d'État totalement laïques et des politiques publiques où la morale ne s'identifie plus avec la morale catholique ou, pour le moins, la morale chrétienne. Les années à venir démontreront si notre analyse s’avère juste.

\author{
Marco Antonio HUACO PALOMINO \\ Lima, Universidad National Mayor de San Marcos \\ marcohuaco@gmail.com
}

\title{
Bibliographie
}

ARMas Asín Fernando, 2000, «Libertad religiosa, violencia y Derechos Humanos en el Perú de fines del siglo XX ", in La Religión en el Perú al Filo del Milenio, Lima, PUCP.

Conferencia Episcopal Peruana, 1988, "Religión e Iglesia en la nueva Constitución », in Bernales Enrique y Rubio M., Constitución: Fuentes e Interpretación, Lima, Mesa Redonda Éditeurs.

Concile Vatican II, 1988, Documents Complets. Declaración Dignitatis Humanae. Sobre la Libertad Religiosa, Lima, Éditions Paulinas.

De Fuenmayor Amadeo, 1974, La libertad religiosa, Pamplone, Éditions Université de Navarre.

Fernández Coronado Ana, 1995, Estado y confesiones religiosas: Un nuevo modelo de relación (Los pactos con las confesiones: Leyes 24, 25 y 26 de 1992), Madrid, Éditions Civitas SA.

García Belaúnde Domingo, 1970, El Constitucionalismo peruano y sus problemas. Selección de textos para el Primer Curso de Derecho Constitucional Peruano, Lima, PUCP, Programme universitaire de Droit.

GaraycoA Hawkins Hugo, 1964, Primeras relaciones entre la Santa Sede y el Perú, Thèse de doctorat, Université Pontificale de Rome.

-, 1965, «El Perú y las tentativas de Concordato », in Derecho, Órgano de la Facultad de Derecho, Lima, PUCP.

Huaco Palomino Marco, 2005, Derecho de la Religión. El principio y derecho de libertad religiosa en el ordenamiento jurídico peruano, Lima, Fonds Éditorial de l'Université Nacional Mayor de San Marcos.

Millot Micheline, 2008, La laïcité, Montréal, Éditions Novalis.

Pareja Paz-Soldán Carlos, 1943, "La necesidad de un Concordato en el Perú », Revue de l'Université Catholique du Pérou, XI, pp. 229-235.

-, 1938, "Las relaciones de la Iglesia y el Estado en la Constitución de 1933 ", Revue de l'Université Catholique du Pérou, VI, pp. 166-184. 


\section{Résumé}

De notre point de vue, le Pérou est passé d'un confessionnalisme doctrinal (où l'État "croit » et "proclame» quelles sont les croyances religieuses légitimes), à un confessionnalisme historico-sociologique. Celui-ci est une forme mitigée de confessionnalisme constitutionnel, caractéristique des transitions historiques vers la lä̈cité, où l'État en tant que tel ne croit pas, ne proclame pas, et ne se considère pas compétent pour déclarer qu'une religion est vraie et que les autres sont fausses, mais privilégie une Église aux dépens des autres pour des raisons historiques (contribution à l'identité nationale) et/ou sociologiques (elle représente la majorité sociale).

Au Pérou, l'histoire des relations entre l'État et les Églises est principalement l'histoire des relations avec l'histoire catholique régies successivement par le Patronat royal (1508-1821), le Patronat républicain de facto (1821-1875), le Patronat républicain de jure (1875-1979) et l'étape actuelle de Concordat (1979 à nos jours). L'argument central de cet article est que depuis 1980, date de la signature d'un accord entre le Saint-Siège et l'État péruvien, ce dernier hésite entre une transition vers un multiconfessionnalisme interreligieux ou vers des formes et des politiques de gouvernement entièrement laïques.

Mots-clés : Laïcité, "Patronat», confessionnalisme, Pérou, minorités religieuses.

\section{Abstract}

In our opinion, Peru has shifted from a doctrinal confessionalism (where the State "believes" and "confesses" a certain religious beliefs as the only genuine), towards a historical-sociological mitigated form of confessionalism which is typical of historic transition to secularism in the State as such does not create or confess, or consider itself competent to declare a particular religion true and others false, but favors a church to the expense of the other due to historical reasons (contribution to national identity) and/or sociological (social majority).

In Peru, the history of relations between State and Church has been largely the history of relations with the Catholic Church, which in turn are reflected by the regional board of trustees (1492-1821), the de facto Republican patronage (18211875), the de jure Republican patronage (1875-1979) and the current concordatary stage (1979-present).

The central thesis of this contribution is that since 1980, an international agreement is concluded between the Holy See and the Peruvian State, an incipient tension exists between moving toward a interreligious multi-confessionalism in the Catholic Church or move toward full political and secular forms of government and State.

Key words: Secularism, "Patronage", confessionalism, Peru, religious minorities.

\section{Resumen}

En nuestra opinión, el Perú ha transitado de una confesionalidad doctrinal (en la que el Estado "cree" y "confiesa" unas determinadas creencias religiosas como únicas verdaderas) hacia una confesionalidad histórico-sociológica, forma mitigada de confesionalidad constitucional que es propia de transiciones históricas hacia la laicidad en la que el Estado como tal no cree ni confiesa, ni se considera competente para declarar que una religión determinada sea verdadera y las otras falsas, aunque 


\section{8 - ARCHIVES DE SCIENCES SOCIALES DES RELIGIONS}

síprivilegie a una iglesia por sobre las otras debido a justificaciones históricas (contribución a la identidad nacional) y/o sociológicas (ser mayoría social).

En el Perú, la historia de las relaciones entre el Estado y las iglesias ha sido mayormente la historia de las relaciones con la Iglesia católica las que se han plasmado sucesivamente mediante el patronato regio (1508-1821), el patronato republicano de facto (1821-1875), el patronato republicano de jure (1875-1979) y la actual etapa concordataria (1979-hoy). La tesis central de la presente contribución es que desde el año 1980 en que se celebra un acuerdo internacional entre la Santa Sede y el Estado peruano, éste se encuentra en una incipiente tensión entre el transitar hacia una pluriconfesionalidad interreligiosa o el avanzar hacia formas y politicas plenamente laicas de gobierno y de Estado.

Palabras clave: Laicidad, "Patronato", confesionalidad, Perú, minorías religiosas. 